\title{
OPTIMIZATION OF THE SPECTRAL RADIUS OF NONNEGATIVE MATRICES
}

\author{
Michael NeUmanN $^{1}$ AND Nung-Sing SzE
}

\begin{abstract}
In a recent paper by Axtell, Han, Hershkowitz, and the present authors, one of the main questions that was considered was finding $n \times n$ doubly stochastic matrices $P$ and $Q$ which solve the multiplicative extremal spectral radius problems $\min _{S \in \Omega_{n}} \rho(S A)$ and $\max _{S \in \Omega_{n}} \rho(S A)$, respectively. Here $A \in \mathbb{R}^{n, n}$ is an arbitrary, but fixed, $n \times n$ nonnegative matrix, $\rho(\cdot)$ is the spectral radius of a matrix, and $\Omega_{n}$ is the set of all $n \times n$ doubly stochastic matrices. It was shown there that the solution to both problems is attained at some permutation matrix. In this paper we consider an additive version of these problems, namely, of solving the additive extremal spectral radius problems $\min _{S \in \Omega_{n}} \rho(S+A)$ and $\max _{S \in \Omega_{n}} \rho(S+A)$. As a by product of, actually, solutions to more general spectral radius optimization problems, we obtain here that the solution to both additive spectral radius optimization problems is, once again, attained at some permutation matrix. One of the more general spectral radius optimization problems that we consider here is that of replacing the constrains that the optimization be done on the doubly stochastic matrices by the weaker constraint of optimizing just on the $n \times n$ column or row stochastic matrices.
\end{abstract}

Mathematics subject classification (2000): 15A48, 15A18. matrices.

Key words and phrases: Nonnegative matrices, spectral radius, doubly stochastic matrices, stochastic

\section{REFERENCES}

[1] J. Axtell, L. Han, D. Hershrowitz, M. Neumann and N. S. Sze, Optimization of the Spectral Radius of a Product for Nonnegative Matrices, Lin. Alg. Appl., to appear.

[2] A. Berman and R. J. Plemmons, Nonnegative Matrices in the Mathematical Sciences, SIAM Publications, Philadelphia, 1994.

[3] J. E. CoHEn, Derivatives of the spectral radius as a function of a non-negative matrix, Math. Proc. Cambridge Philos. Soc., 83 (1978), 183-190.

[4] E. Deutsch AND M. Neumann, Derivatives of the Perron root at an essentially nonnegative matrix and the group inverse of an M-matrix, J. Math. Anal. Appl., 102 (1984), 1-29.

[5] L. ELSNER, On convexity properties of the spectral radius of nonnegative matrices, Lin. Alg. Appl., 61 (1984), 31-35.

[6] S. FrIEdLAND, Convex spectral functions, Lin. Multilin. Alg, 9 (1981), 299-316.

[7] G. H. GOLUB AND C. D. MEYER, JR., Using the QR-factorization and group inversion to compute, differentiate, and estimate the sensitivity of stationary probabilities for Markov chains, SIAM J. Alg. Discrete Methods, 7 (1986), 273-281.

[8] L. HAN, M. NEUMANN AND M. J. TSATSOMEROS, Spectral radii of fixed Frobenius norm perturbations of nonnegative matrices. SIAM J. Matrix Anal. Appl., 21 (1999), 79-92.

[9] C. R. JOHNSON, R. LOEWY, D. D. OleSKY AND P. VAN DEN DRIESSCHE, Maximizing the spectral radius of fixed trace diagonal perturbations of nonnegative matrices, Lin. Alg. Appl., 241-243 (1996), 635-654. 